\title{
Application of RMAN Backup Technology in the Agricultural Products Wholesale Market System
}

\author{
Ping $\mathrm{Yu}^{1,2}$ and Nan $\mathrm{Zhou}{ }^{1}$ \\ ${ }^{1}$ Network Center, China Agricultural University, Beijing 100083, China \\ ${ }^{2}$ School of Economics \& Management, China Agricultural University, Beijing 100083, China \\ \{yuping, zhnan\}@cau.edu.cn
}

\begin{abstract}
Based on Oracle Database RMAN backup technology, this paper designs and implements a database backup subsystem for the agricultural products wholesale market system. Through full backup, incremental backups and cumulative incremental backup, it ensures the timeliness, integrity and efficiency of data recovery, when data mistake happens in the agricultural products wholesale market system database or the database collapses.
\end{abstract}

Keywords: agricultural products wholesale market system, Oracle database backup, RMAN.

\section{$1 \quad$ Introduction}

The development of computer technology and network technology makes database technology to be widely applied in enterprise management information systems, ecommerce systems, e-government systems and other application systems. With 随the continuous propelling of informatization, the amount of data in application systems grows sustainably. Data security issue in database systems have become increasingly important.

RMAN is Oracle tools which is used to backup, restore and restore Oracle database. RMAN can only be used in ORACLE8 or later versions. Researchers have applied Oracle backup technology to different areas. ZHANG Yun-fan discusses the application of backup and recovery technology of Oracle database in oil field exploration project [1]. Feng ke et al. gives a brief summary for database administrators who pay more attentions to the usability of backup data usability and auto-monitoring technique, the technique is applied in database backup of university and proved to be effective [2]. ZHU Youcun et al. realized rapid backup and recovery based on oracle 10 and applied it in the database of hospital [3]. Following the database backup technology of Daqing logging production database wag still in the stage of the logical export manually and has not yet had an effective data backup technology to ensure data security, LIU Yubin uses RMAN backup technology on the features of the production database to deploy a perfect backup program in practice [4] .

The agricultural products wholesale market system database has stored the price information and quality testing data of agricultural products. The safe storage of these data plays a crucial role in price management, quality inspection and macro decisionmaking of the agricultural products wholesale market. Therefore, when it appears that 
data is accidentally deleted or database crashes, the requirement for its data recovery is zero-loss and to recovery to a designated time point. In addition, as the amount of data in this database is very large, and the allowed downtime of this system is 10 minutes, it requires the backup and recovery efficiency is as high as possible. Common logical backup will cause some data cannot be recovered, and the recovery time is far from the actual needs, therefore it needs to design a real-time, fully synchronized and strategic reasonable data backup subsystem.

RMAN can back up the entire database or database components, such as table spaces, data files, control files, archive files, and Spfile parameter file [5]. RMAN also allows you to conduct block-level incremental backup, incremental RMAN backups are time and space efficient because they only backup those data blocks which have changed since the last backup [6]. Therefore, it can well meet the data recovery needs of the agricultural products wholesale market system, whether from the respect of real-time or recovery efficiency.

In this paper, we used Oracle Database RMAN backup technology to design and implement a backup subsystem for the agricultural products wholesale market system.

\section{Agricultural Products Wholesale Market System}

The agricultural products wholesale market system is the first phase of Golden Agriculture Project, it uses the agricultural products wholesale market as the main datasource to collect the prices and the quality inspection data of agricultural products, it goes through data collection segment, the data processing segment, data

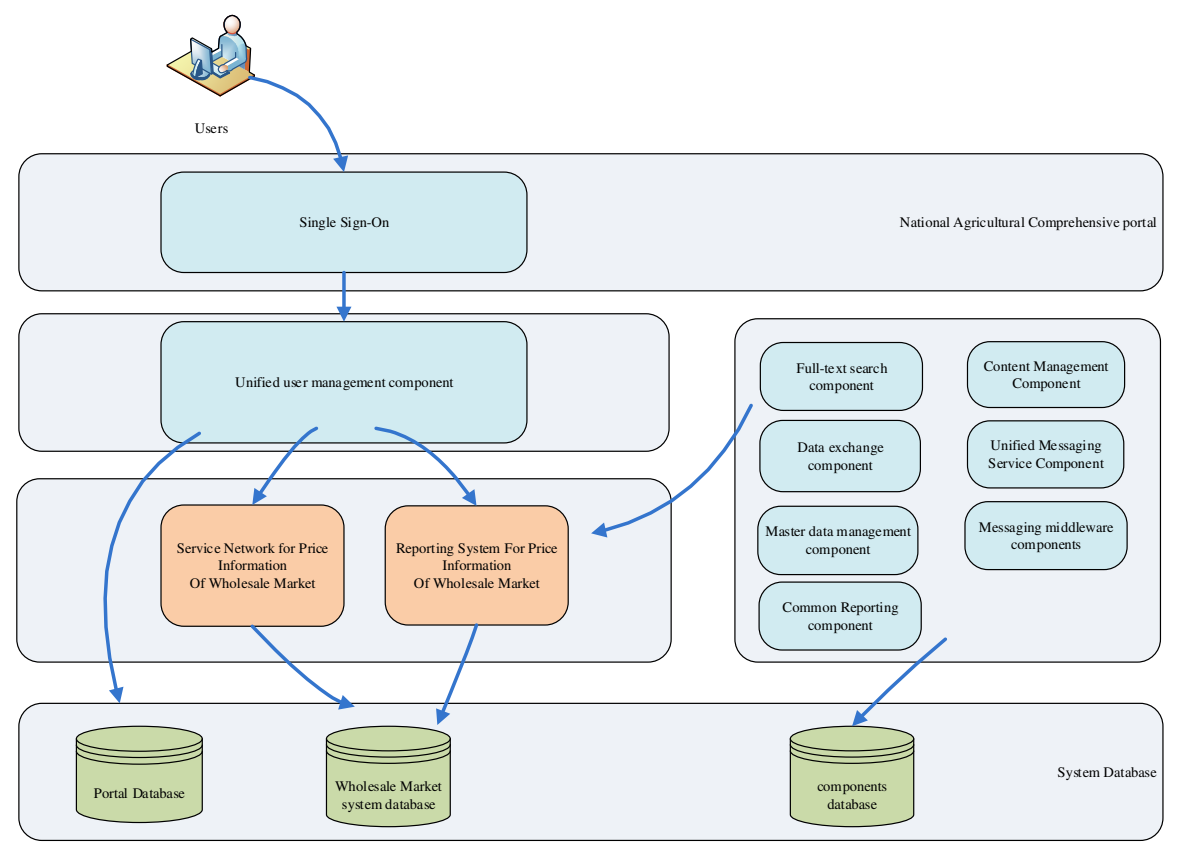

Fig. 1. The technical architecture of the agricultural products wholesale market system 
analysis and display segment. The technical architecture of the agricultural products wholesale market system is shown as Fig.1.

As shown in Figure 1, System database includes portal database, the wholesale market system database and components database, it is an important support for the running of the agricultural product wholesale market system, as all of the system's data changes have been recorded in the database, if these data is lost, it will directly affect the normal running of the agricultural products wholesale business, so how to ensure data not to be lost, is essential. So it is important to choose a reasonable backup mode and develop a reasonable backup strategy to design and implement the agricultural products wholesale market system backup subsystem, so as to ensure the data not to be lost.

We choose Oracle10g for windows to meet the system's requirement for the capacity, response speed, database security, stability and scalability of database.

\section{Database Backup Subsystem Based on RMAN Technology}

\subsection{The Architecture of RMAN Technology}

The architecture of RMAN can be expressed in Fig.2 [7].

Through the above architecture diagram, we can know oracle RMAN is actually through the queries on the basis table of target database and the system views to conduct online hot backup for the database data files ,control files and spfile files and

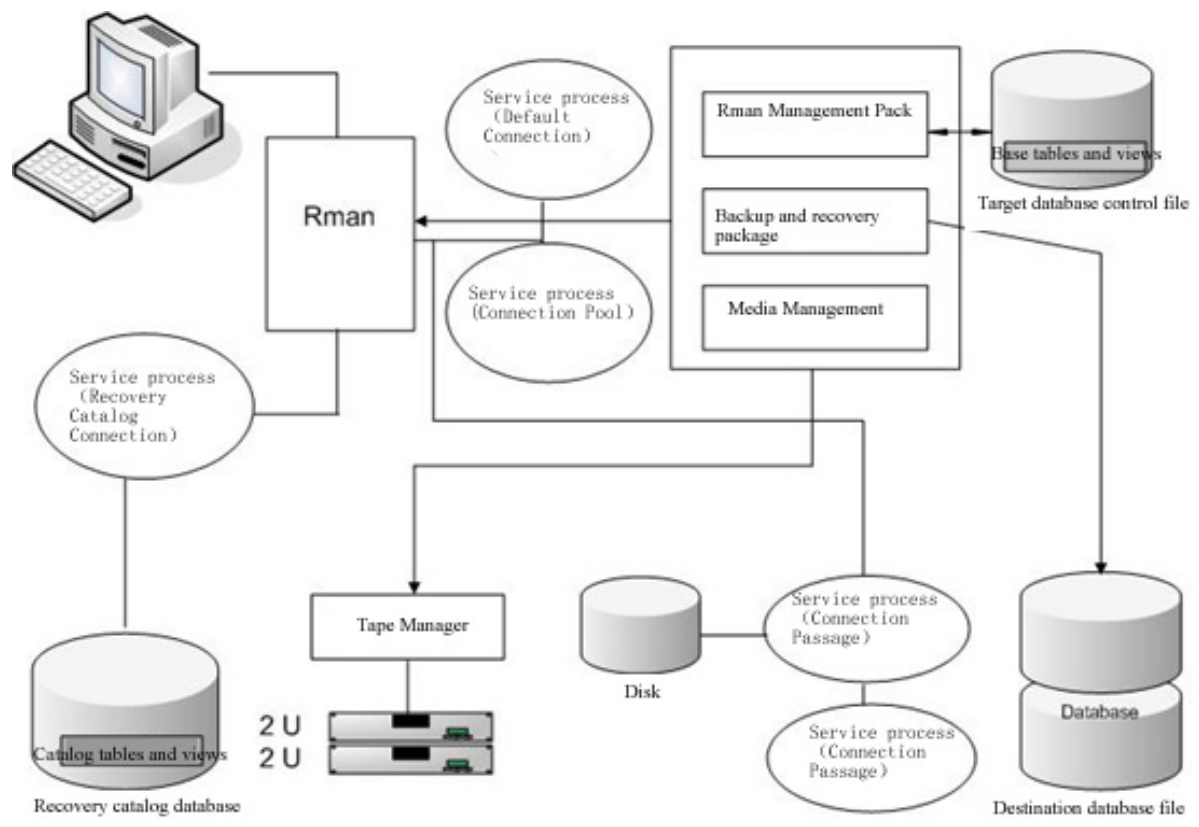

Fig. 2. The architecture of RMAN 
so on, so as to achieve the recovery of target database after a crash [8]. In addition, in order to avoid bringing the performance loss to the operating system by backing up all files in each backup, RMAN also provides a backup of the archive logs, so that we can develop an appropriate strategy, namely conducting incremental archive logs backup after full backup. When the database crashes, we first restore full backup, and then apply the incremental backup archive to achieve full recovery or the specified point in time recovery. At backup time, RMAN will store the backup information of each time in the recovery catalog database, and establish a connection channel, and then through media management, transfer the backup sets to the backup media. When restoring database, RMAN will search in the recovery catalog database according to our specified conditions, when it finds the corresponding backup piece, it will use the oracle recovery package to conduct the corresponding recovery.

\subsection{The Design of Database Backup Subsystem}

By analyzing the characteristics of the agricultural products wholesale market system, we know that the amount of data in the database will be very large, and every Monday to Friday is the peak of the business, the data volume has increased significantly more than on Saturday and Sunday; the importance of its data is very high, it does not allow missing data and its recovery time should be as short as possible. Therefore, its backup strategy is as follows: Choose full backups on Sunday to reduce impact of full backups on the database performance; the data recovery on Monday, Tuesday will be completed through the full backups on Sunday and the incremental backup on the two days; in order to improve the recovery speed, choose to conduct cumulative incremental backups on Wednesday, the recovery on Wednesday will be completed through the full backups on Sunday and the cumulative incremental backups on Wednesday; the recovery on Thursday, Friday and Saturday will be completed by the full backups on Sunday, the cumulative incremental backups on Wednesday and incremental backups on Thursday, Friday and Saturday. The specific backup strategy is shown in Fig.3.
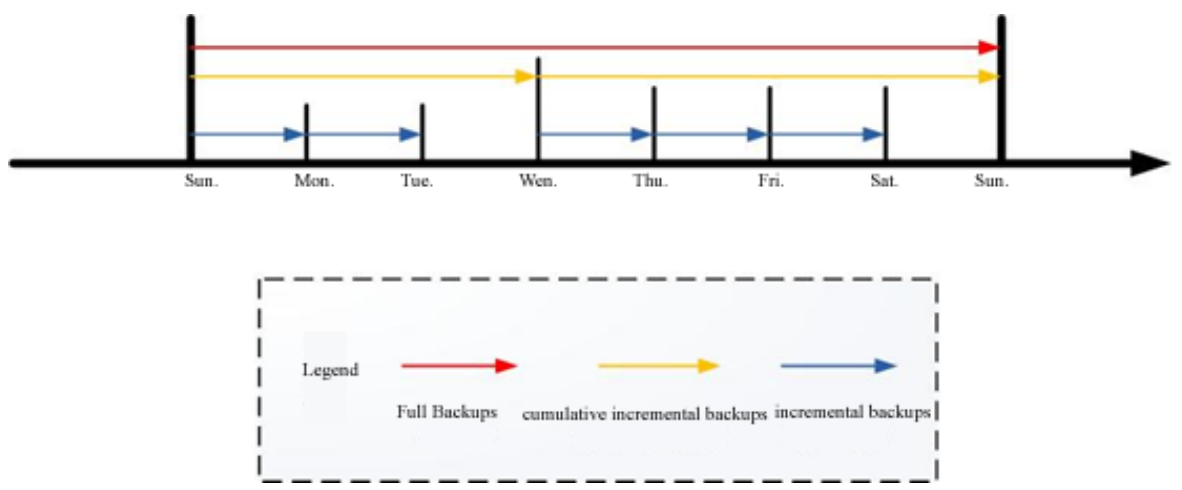

Fig. 3. Backup strategy of database backup subsystem 


\subsection{The Implementation of Database Backup Subsystem}

1. Application Preparation

Before using RMAN backup for the first time, we need to do some preparatory work, including set the target database to archive database, because RMAN needs the

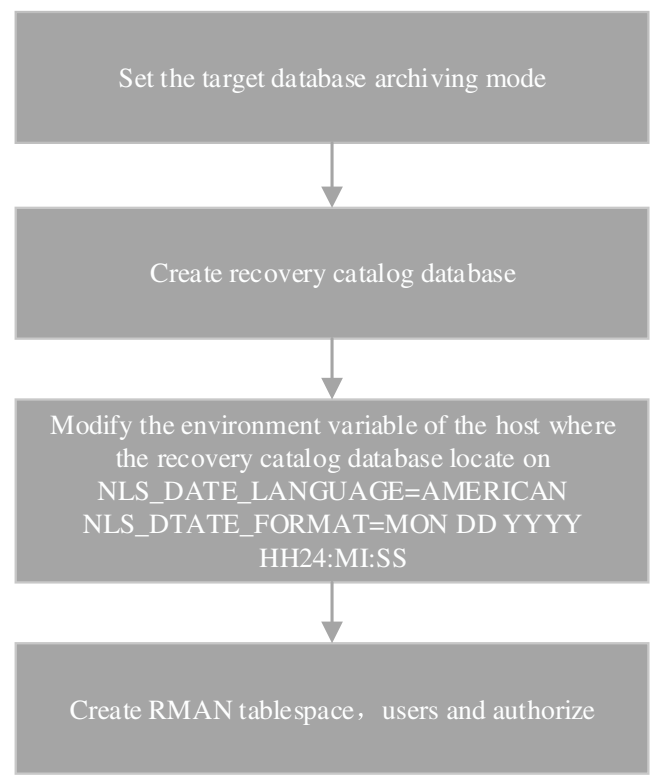

Fig. 4. Preparation before application

archive log file support when doing the time-based recovery or the full recovery or other recoveries. In addition, we need to create a database and create a table space and user in the database to store the recovery catalog information. These works can be broadly described in Fig.4.

2. The design and implementation of backup and recovery program

The full backup program on every Sunday are summarized as below: run \{

allocate channel 'dev1' type disk ;

allocate channel 'dev2' type disk;

allocate channel 'dev3' type disk ;

allocate channel 'dev4' type disk ;

backup incremental level 0 tag 'dbL0' format '<path>llevel0_\%u_\%p_\%c' database skip readonly;

sql 'alter system archive log current' ;

backup archivelog all delete input;

release channel dev1;

release channel dev2;

release channel dev3; 
release channel dev4;

\}

The incremental backup program on Monday, Tuesday, Thursday, Friday and Saturday are summarized as below:

run \{

allocate channel 'dev1' type disk ;

allocate channel 'dev2' type disk ;

allocate channel 'dev3' type disk ;

allocate channel 'dev4' type disk ;

backup incremental level 2 cumulative tag 'dbL2' format '<path>/level2c_\%u_\%p_\%c' database skip readonly;

sql 'alter system archive log current' ;

backup archivelog all delete input;

release channel dev1;

release channel dev2;

release channel dev3;

release channel dev4;

\}

The incremental backup program on Wednesday are summarized as below: run \{

allocate channel 'dev1' type disk ;

allocate channel 'dev2' type disk ;

allocate channel 'dev3' type disk ;

allocate channel 'dev4' type disk ;

backup incremental level $1 \mathrm{tag}$ 'dbL2' format '<path>/level2_\%u_\%p_\%c' database skip readonly;

sql 'alter system archive log current' ;

backup archivelog all delete input;

release channel dev1;

release channel dev2;

release channel dev3;

release channel dev4;

\}

These backup program are set to be executed through the timed job system of windows.

3. Backup and recovery joint adjusting

After making these preparations, we can officially start using RMAN for data backup. This includes running RMAN Manager to connect to the target database and recovery catalog database, register the target database, synchronize the information between the target database, the directory database, and the backup pieces, distribute and establish channels to a connection, run the script for backup and recovery, and finally release the connecting channels and other steps. These steps can be simplified represented in Fig.5. 


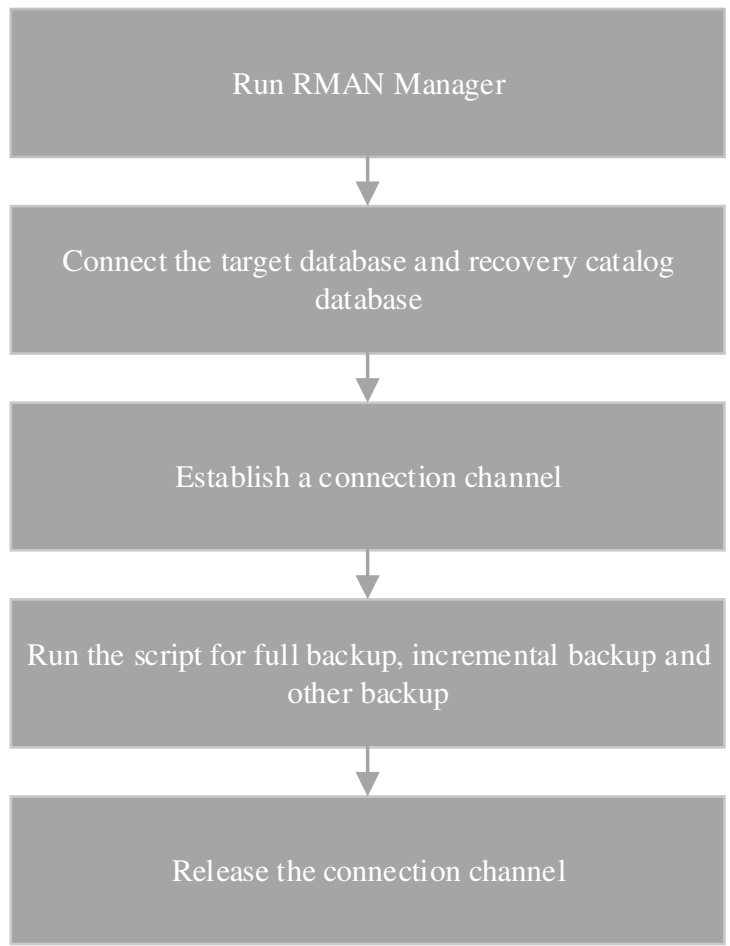

Fig. 5. The backup and recovery processes of RMAN

\section{$4 \quad$ Test and Run}

The agricultural products wholesale market system was officially launched in the end of December 2011, its database system was Oracle 10G installed under WINDOWS, and Oracle practical cluster systems was installed to ensure the high availability of the database. The physical structure of its operating environment is shown as Fig.6.

As can be seen from the figure, the database realized Oracle utilities (RAC) via two IBM p690 cluster systems. When there is a data error operation, the data can be restored through the database backup system. 


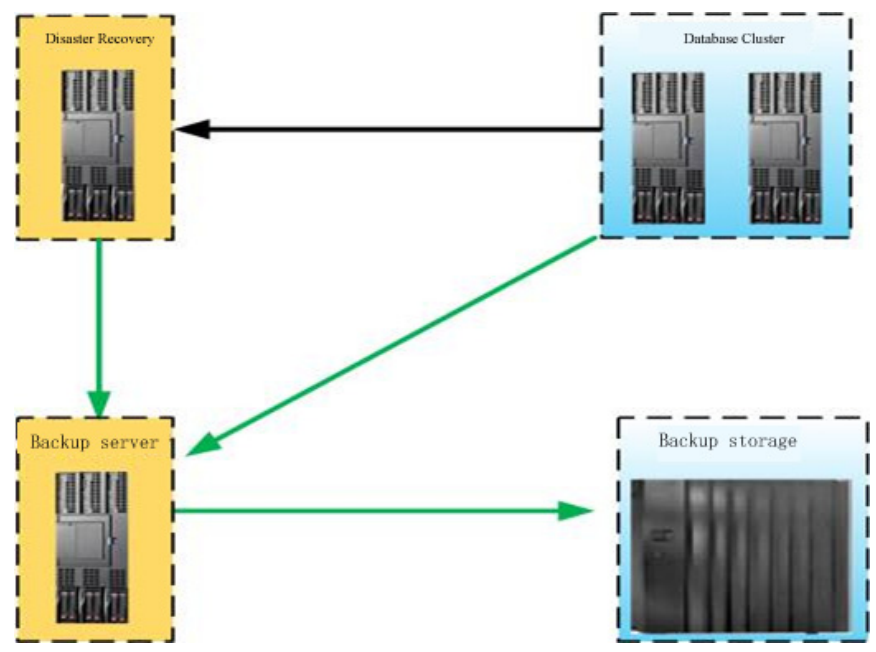

Fig. 6. The structure of database backup subsystem

After two weeks of installation and commissioning, the data backup functionality has been achieved, and in 2012 we conducted a backup and recovery test, which successfully implement the full database restore and the recovery based on the specified point in time, and ensured zero data loss and the recovery of accidentally deleted data.

\section{Conclusion}

This paper designed and implemented a database backup subsystem based on the Oracle Database RMAN backup technology, and successfully deployed it into the agricultural products wholesale market system, which ensures the security, reliability and recoverability of the back-end database of the system. It is also able to adapt to the security requirement of the slimily system.

\section{References}

1. Zhang, Y.-F.: Backup and Recovery Strategy of Oracle Database. Computer Engineering 35(15), 85-87 (2009)

2. Feng, K., Liu, N.-J., Wang, Q., Li, T.: Research and practice of auto-monitoring for Oracle database backup. Experimental Technology and Management 28(4) (2011)

3. Zhu, Y.-C., Luo, D., Yang, X.-R.: RMAN Backup, Recovery and optimization Based on oracle 10g. Medical Equipment 33(10), 45-46 (2012)

4. Liu, Y.-B.: The Research and Implementation of Database Backup Program Based on Oracle. Programming Skills \& Maintenance 14, 025 (2009)

5. Freeman, R.G., Hart, M.: Oracle9i RMAN backup \& recovery. McGraw-Hill/Osborne (2002)

6. Hart, M., Freeman, R.G.: Oracle Database 10g RMAN Backup and Recovery (2007)

7. Oracle RMAN Pocket Reference. O'Reilly Media, Inc. (2009)

8. Alapati, S.R., Kuhn, D., Nanda, A.: RMAN recipes for Oracle database 11g: A problemsolution approach. Apress (2007) 